\title{
The features of application of different systems of orientation angles at the processing of aerospace images
}

\author{
Vladimir Bezmenov \\ Kazan Federal University, Department of Astronomy and Space Geodesy, 420008 Kremlin str, \\ Kazan, Russian Federation
}

\begin{abstract}
The angular elements of external orientation characterize the position of a shooting camera relative to the coordinate system in which the spatial coordinates of the points of the object under study are determined from the processing of its images. In many cases of aerial photography, e.g. shooting from an unmanned aerial vehicle, as well as in the case of space survey, the values of the orientation angles could be very significant. This paper presents the results of numerical experiments for five different systems of external orientation angles (Euler angles). The studies were performed using the condition of complanarity, which is the basis of space forward intersection. For a space forward intersection, a model of errors in determining spatial coordinates for five systems of shooting camera orientation angles has been developed. In the numerical experiments, the general case of aerial photography from an unmanned aerial vehicle and of space survey of the Earth were simulated. By comparing the root-mean-square errors (RMSE) in determining the spatial coordinates obtained using the studied systems of external orientation angles, the features of the use of these systems of orientation angles were revealed. The results of the research will allow to determine the spatial coordinates of the points of the studied objects with a greater degree of reliability by photogrammetry methods.
\end{abstract}

Keywords: external orientation angles, space forward intersection, spatial coordinates, root-mean-square errors, unmanned aerial vehicles, remote sensing.

\section{Introduction}

In many cases, taking into account the development of modern technologies and the tasks being solved, the angular elements of the external orientation of the shooting camera may have large values and be in the range from $0^{\circ}$ to $360^{\circ}$ :

- in ground-based survey as well as in aerial survey of buildings and structures using unmanned aerial vehicles (UAVs) in order to build a 3D model and when calculations are performed in the coordinate system of the corresponding region;

- in space survey and aerial survey, when the geocentric coordinate system is used as an external coordinate system in which the angular position of the image (camera) is characterized [1]. 
The relevance of the topic considered in the article is also caused by the trend of modern photogrammetric technologies aimed at determining the spatial coordinates of the set of points of the object under study without using reference points: only based on the initial information georeferenced with the required accuracy, i.e. images. For example, in the article [2] concerning the issues of ensuring the geographic referencing of remote sensing of the Earth (RSE) images, the aspects as follows are noted:

- one of the most important characteristics of RSE satellite data is the accuracy of their georeferencing [2];

- it is possible to provide georeferencing on the basis of readings of onboard measuring instruments only, without using reference points on the terrain.

One of the components of georeferencing are orientation angles of the camera. The influence of the orientation angles on the final error in determining the spatial coordinates is significant and may be decisive [2, 3, 4].

The question of the influence of systems of orientation angles on the error (accuracy) in determining the spatial coordinates of points of the terrain (object) is considered in [1]. This article presents the research results for two systems of exterior orientation angles:

- system of $\alpha, \omega, \chi$ angles (longitudinal and transverse tilt angles, image rotation angle), referred to in the Russian-language literature as the second system of angles;

- system of $\bar{\omega}, \varphi, \kappa$ angles (transverse and longitudinal tilt angles, image rotation angle), recommended for use by the International Society of Photogrammetry and Remote Sensing (ISPRS).

The article [1], in particular, notes:

- for plane aerial survey, when the tilt angles are small, both variants of the external orientation angular elements are equal, and the results of solving photogrammetric problems will coincide up to round-off errors;

- in the case when the angular elements will have larger values, the choice of the external orientation system may be decisive and the angular orientation system should be selected taking into account the position of the images relative to the external coordinate system of the terrain or the photographed object.

The combination of several systems of external rectangular coordinates for different parts of the object, selected so that the angles of inclination of the images lie within the required limits, as suggested in [1], cannot be an effective technology, since it requires additional actions to form a general model of the object under study and will contribute to the deformation of the model.

In addition to the systems of orientation angles $\alpha, \omega, \chi$ and $\bar{\omega}, \varphi, \kappa$ considered in [1], several more systems of external orientation angles (Euler angles) are known, which are used in the theory and practice of photogrammetry and remote sensing $[5,6,7,8]$ :

- system of $\varphi, \tau, \psi$ angles used in aviation (roll, pitch and yaw angles);

- system of $t, \alpha_{0}, \chi$ angles referred to in the Russian-language literature as the first system of angles (directional angle and tilt angle, optical axis angle, image rotation angle);

- system of $\Omega, J, \omega$ angles used in space photogrammetry (similar to the angles used in celestial mechanics).

The question of the influence of orientation angles systems on the error (accuracy) of determining the spatial coordinates of points of the terrain (object) could be considered not only based on the use of the collinearity condition as it is done in [1]. This article provides a comparative analysis of the influence of the five listed systems of orientation angles $-\alpha, \omega$, $\chi ; \bar{\omega}, \varphi, \kappa ; t, \alpha_{0}, \chi ; \varphi, \tau, \psi$ and $\Omega, J, \omega-$ on the error (accuracy) of determining spatial coordinates. The studies were carried out using the complanarity condition, which is the basis for space forward intersection. 


\section{Mathematical foundations for solving the problem}

Euler angles $\left(\alpha, \omega, \chi ; \bar{\omega}, \varphi, \kappa ; \varphi, \tau, \psi ; t, \alpha_{0}, \chi ; \Omega, J, \omega\right)$ allow determining the final matrix of orthogonal transformations corresponding to 3 elementary rotations and characterizing the transformation from the coordinate system of the image space $\tilde{x}, \tilde{y}, \widetilde{z}-$ a coordinate system with the origin at the center of the projection of the shooting camera - to the coordinate system of the object space $X, Y, Z$ :

$$
A=A_{\alpha \omega \chi}=A_{\bar{\omega} \varphi \kappa}=A_{\psi \tau \varphi}=A_{t \alpha_{0} \chi}=A_{\Omega J \tau} .
$$

The resulting rotation matrices for the listed systems of angles will be calculated by the formulas:

$$
\begin{gathered}
A_{\alpha \omega \chi}=A_{\alpha}^{2 \uparrow} A_{\omega}^{1 \downarrow} A_{\chi}^{3 \downarrow} ; A_{\bar{\omega} \varphi \kappa}=A_{\vec{\omega}}^{1 \downarrow} A_{\varphi}^{2 \downarrow} A_{\kappa}^{3 \downarrow} ; A_{\psi \tau \varphi}=A_{\psi}^{3 \downarrow} A_{\tau}^{2 \downarrow} A_{\varphi}^{1 \uparrow} ; \\
A_{t \alpha 0 \chi}=A_{t}^{3 \downarrow} A_{\alpha_{0}}^{2 \uparrow} A_{\chi}^{3 \downarrow} ; A_{\Omega J \omega}=A_{\Omega}^{3 \downarrow} A_{J}^{1 \downarrow} A_{\omega}^{3 \downarrow} .
\end{gathered}
$$

In formulas (2) and (3), the following designations are introduced: $A_{\theta}^{i \downarrow}, A_{\theta}^{i \uparrow}$ - matrices of orthogonal transformations corresponding to elementary rotation around the $i$-th $(i=1,2,3)$ axis of the spatial coordinate system $X Y Z$ by some angle $\theta$, clockwise ( $\uparrow$ ) or counterclockwise $(\downarrow)$. The formulas for calculating the direction cosines $a_{\mathrm{kl}}(k, 1=1,2,3)$ of the final rotation matrices corresponding to the listed systems of angles are known and, in particular, are given in $[1,6,7,8,10]$. It is known that the determination of the values of the orientation angles using the direction cosines leads to the uncertainty of a pair of angles. In the listed systems of orientation angles, such an uncertainty will be as follows:

in the system of $\alpha, \omega, \chi$ angles - for $\alpha$ and $\chi$, at $\omega=90^{\circ}$;

in the system of $\bar{\omega}, \varphi, \kappa$ angles - for $\bar{\omega}$ and $\kappa$ at $\varphi=90^{\circ}$;

in the system of $\varphi, \tau, \psi$ angles - for $\varphi$ and $\psi$ at $\tau=90^{\circ}$;

in the system of $t, \alpha_{0}, \chi$ angles - for $t$ and $\chi$ at $\alpha_{0}=90^{\circ}$;

in the system of $\Omega, J, \omega$ angles - for $\Omega$ and $\omega$ at $J=90^{\circ}$.

Determination of angles using direction cosines has the property of ambiguity, which is expressed in the impossibility of determining the quarters in which the angles of the camera tilt are located. Ambiguity does not appear in the case of a plane survey and when the angles $\omega, \varphi, \tau, \alpha_{0}, J$ in absolute value are less than $90^{\circ}$. The ambiguity property becomes very important when determining the external orientation angles by the method of the inverse photogrammetric problem, i.e. when using control points, in the case of significant dimensions of the photogrammetric construction, when the absolute values of the marked angles are greater than $90^{\circ}$. Elimination of this ambiguity through the calculation of the elements of mutual orientation [1] cannot be considered an effective approach, especially in the information processing mode close to the real-time one. A more correct and effective solution to this issue, both in the case of imaging from a UAV and from an RSE spacecraft, is to determine the orientation angles: the angles $\varphi, \tau, \psi$ (roll, yaw and pitch) directly in flight. The calculation of other systems of angles will be performed unambiguously and can be verified by modeling for a specific image. In work [1], in relation to the angles of orientation $\alpha, \omega, \chi$ and $\bar{\omega}, \varphi, \kappa$, it is noted that the poor conditionality of the matrix of normal equations of the space inverse intersection at angles $\omega \approx \varphi \approx 90^{\circ}$, leading to significant errors in determining the angles, is not necessarily reflected in observance of the collinearity condition and on the coordinates of the photographing centers. At the same time, it is quite rightly noted that a space forward intersection will provide more correct 
coordinates of terrain points.

The studies performed in this article is based on the use of a multiparameter model of root mean square errors (RMSEs) for spatial coordinates determined by the method of space forward intersection (SFI) for an arbitrary shooting case [9]. The parameters of this model, in particular, are linear and angular exterior orientation elements of a pair of images. There are 19 parameters of the model $(i=1,2, \ldots, 19)$. The general model is quite universal, taking into account the peculiarities in the calculation of some coefficients, which is caused by the applied system of orientation angles. Therefore, in this article, in order to preserve generality in the research, the designations of the parameters that were used in the article [9] are applied.

The solution to the problem of estimating the SFI accuracy, available for 3 systems of exterior orientation angular elements $-\alpha, \omega, \chi ; \bar{\omega}, \varphi, \kappa ; \Omega, J, \omega-$ was expanded to 5 systems, including systems of orientation angles $\varphi, \tau, \psi$ and $t, \alpha_{0}, \chi$. In the numerical experiments, modeling of aerial and space survey using the systems of angles $\varphi, \tau, \psi$ and $t$, $\alpha_{0}, \chi$ was carried out according to the formulas given in [9], taking into account the following established features for the coefficients included in them:

1. The coefficients $A_{\mathrm{i}}, B_{\mathrm{i}}, E_{\mathrm{i}}$ for the system of angles $t, \alpha_{0}, \chi(i=1,2)$ and the system of angles $\varphi, \tau, \psi(i=1,2,3)$ will be calculated by the formulas listed in the Table 1 and Table 2. The rest of the coefficients $A_{\mathrm{i}}, B_{\mathrm{i}}, E_{\mathrm{i}}$ will be determined by the formulas given in the article [9].

2. The calculation of the $Q_{\mathrm{i}}$ values, which are partial derivatives of the parallax coefficient $N$ by the parameters included in the RMSE model, will be carried out using general formulas for any systems of orientation angles [9]. The values $L_{\mathrm{i}, \mathrm{j}}$ and $K_{\mathrm{i}, \mathrm{j}}(i=1,2,3, \ldots, 19$; $j=1,2,3)$ used in the formulas for $\mathrm{Q}_{\mathrm{i}}$ have singularities for each system of angles. These features are present in the first $6 L_{\mathrm{i}, \mathrm{j}}$ and $K_{\mathrm{i}, \mathrm{j}}$. For a system of angles $t, \alpha_{0}, \chi$ and a system of angles $\varphi, \tau, \psi$, this will be, respectively, for $i=1,2,4,5$ and $i=1,3,4,6$. Formulas for calculating these $L_{\mathrm{i}, \mathrm{j}}$ and $K_{\mathrm{i}, \mathrm{j}}$ are shown in Table 3 and Table 4.

The parameters given in Table 1, Table 2, Table 3 and Table 4 have the following semantic meaning [9]:

$X_{1}^{\prime}, Y_{1}^{\prime} Z_{1}^{\prime}$ and $X_{2}^{\prime}, Y_{2}^{\prime}, Z_{2}^{\prime}$ - coordinates of the point image on the first (left) and second (right) images in the coordinate system with the origin in the center of the image projection taking into account the orthogonal rotation matrix;

$X_{0}, Y_{0}, Z_{0}$ - components of the vector that determines the position of the right center of the projection relative to the left;

$P_{\mathrm{S}}$ and $\hat{P}_{\mathrm{S}}(s=1,2,3,4,5,6,7)-$ parameters that are also determined for the first and second images, respectively, using the values of the measured coordinates $(x, y)$ of a point in the image, focal length $f$ and orientation angles of the shooting camera.

Table 1. Formulas for calculating the coefficients $A_{\mathrm{i}}, B_{\mathrm{i}}, E_{\mathrm{i}}$ for the angles $\alpha_{0}, t, \chi$.

\begin{tabular}{|c|c|c|c|}
\hline$i$ & $A_{\mathrm{i}}$ & $B_{\mathrm{i}}$ & $E_{\mathrm{i}}$ \\
\hline 1 & $X_{1}^{\prime} Q_{1}+N P_{2}$ & $Y_{1}^{\prime} Q_{1}+N P_{3}$ & $Z_{1}^{\prime} Q_{1}+N P_{4}$ \\
\hline 2 & $X_{1}^{\prime} Q_{2}-N Y_{1}^{\prime}$ & $Y_{1}^{\prime} Q_{2}+N X_{1}^{\prime}$ & $Z_{2}^{\prime} Q_{2}$ \\
\hline
\end{tabular}


Table 2. Formulas for calculating the coefficients $A_{\mathrm{i}}, B_{\mathrm{i}}, E_{\mathrm{i}}$ for the angles $\varphi, \tau, \psi$.

\begin{tabular}{|c|c|c|c|}
\hline$i$ & $A_{\mathrm{i}}$ & $B_{\mathrm{i}}$ & $E_{\mathrm{i}}$ \\
\hline 1 & $X_{1}^{\prime} Q_{1}+N P_{5}$ & $Y_{1}^{\prime} Q_{1}+N P_{6}$ & $Z_{1}^{\prime} Q_{1}+N P_{7}$ \\
\hline 2 & $X_{1}^{\prime} Q_{2}+N P_{2}$ & $Y_{1}^{\prime} Q_{2}+N P_{3}$ & $Z_{1}^{\prime} Q_{2}+N P_{4}$ \\
\hline 3 & $X_{1}^{\prime} Q_{3}-N Y_{1}^{\prime}$ & $Y_{1}^{\prime} Q_{3}+N X_{1}^{\prime}$ & $Z_{1}^{\prime} Q_{3}$ \\
\hline
\end{tabular}

For the system of angles $\alpha_{0}, t, \chi$, the parameters $P_{\mathrm{S}}$ and $\hat{P}_{\mathrm{S}}(s=2,3,4,5,6,7)$ will be calculated by the formulas given in [9]. The calculation of $P_{1}$ and $\hat{P}_{1}$ will be performed by the formula:

$$
P_{1}=X^{\prime}=a_{11} x+a_{12} y-a_{13} f .
$$

The calculation of $\hat{P}$ involves the direction cosines $\hat{a}_{l k}$ of the matrix $\hat{A}$, the coordinates of the image, the value of the focal length for the second image.

For the system of angles $\varphi, \tau, \psi$, the parameters $P_{1}$ and $\hat{P}_{1}$ will be calculated by the formula (4). To calculate $P_{S}$ and $\hat{P}_{S}(s=5,6,7)$, one uses the formulas as follows:

$$
P_{5}=-a_{13} y-a_{12} f ; \quad P_{6}=-a_{23} y-a_{22} f ; \quad P_{7}=-a_{33} y-a_{32} f
$$

The calculation of $P_{\mathrm{S}}$ and $\hat{P}_{\mathrm{S}}(s=2,3,4)$ will be performed according to the formulas given in [9]. The matrix $C$ [9], whose coefficients are used in calculating $P_{\mathrm{s}}$ and $\hat{P}_{\mathrm{S}}$, in the case of a system of angles $t, \alpha_{0}, \chi$ and $\varphi, \tau, \psi$ is the matrix of partial derivatives of matrix $A$ with respect to the angle $\alpha_{0}$ and $\tau$, respectively:

- for $t, \alpha_{0}, \chi$

$$
C=\frac{\partial}{\partial \alpha_{0}} A=\left[\begin{array}{lll}
c_{11} & c_{12} & c_{13} \\
c_{21} & c_{22} & c_{23} \\
c_{31} & c_{32} & c_{33}
\end{array}\right]=\left[\begin{array}{lll}
a_{13} \cos \chi & -a_{13} \sin \chi & -a_{33} \cos t \\
a_{23} \cos \chi & -a_{23} \sin \chi & -a_{33} \sin t \\
a_{33} \cos \chi & -a_{33} \sin \chi & -a_{13} \sin \alpha_{0}
\end{array}\right] ;
$$

- for $\varphi, \tau, \psi$

$$
C=\frac{\partial}{\partial \tau} A=\left[\begin{array}{lll}
c_{11} & c_{12} & c_{13} \\
c_{21} & c_{22} & c_{23} \\
c_{31} & c_{32} & c_{33}
\end{array}\right]=\left[\begin{array}{ccc}
a_{31} \cos \psi & a_{32} \cos \psi & a_{33} \cos \psi \\
a_{31} \sin \psi & a_{32} \sin \psi & a_{33} \sin \psi \\
-\cos \tau & -a_{31} \sin \varphi & a_{31} \cos \varphi
\end{array}\right] .
$$


Table 3. Formulas for calculating $K_{\mathrm{i}, \mathrm{j}}$ и $L_{\mathrm{i}, \mathrm{j}}$ for $\alpha_{0}, t, \chi$ system of angles.

\begin{tabular}{|c|c|c|c|c|c|}
\hline$i$ & $Q_{\mathrm{i}}$ & $\begin{array}{c}L_{\mathrm{i}, \mathrm{j}} \\
K_{\mathrm{i}, \mathrm{j}}\end{array}$ & $j=1$ & $j=2$ & $j=3$ \\
\hline 1 & $Q_{1}$ & $K_{1, \mathrm{j}}$ & $Y_{2}^{\prime} P_{4}-Z_{1}^{\prime} P_{2}$ & $Z_{2}^{\prime} P_{2}-X_{2}^{\prime} P_{4}$ & $X_{2}^{\prime} P_{3}-Y_{2}^{\prime} P_{2}$ \\
\hline 2 & $Q_{2}$ & $K_{2, \mathrm{j}}$ & $-Z_{2}^{\prime} X_{1}^{\prime}$ & $-Z_{2}^{\prime} Y_{1}^{\prime}$ & $Y_{2}^{\prime} Y_{1}^{\prime}+X_{2}^{\prime} X_{1}^{\prime}$ \\
\hline \multirow{2}{*}{4} & \multirow{2}{*}{$Q_{4}$} & $L_{4, \mathrm{j}}$ & $Y_{0} Y_{2}^{\prime}-Z_{0} \hat{P}_{1}$ & $-X_{0} Y_{2}^{\prime}$ & $X_{0} \hat{P}_{1}$ \\
\cline { 3 - 6 } & $K_{4, \mathrm{j}}$ & $Z_{1}^{\prime} \hat{P}_{1}-Y_{1}^{\prime} Y_{2}^{\prime}$ & $X_{1}^{\prime} Y_{2}^{\prime}$ & $-X_{1}^{\prime} \hat{P}_{1}$ \\
\hline \multirow{2}{*}{5} & \multirow{2}{*}{$Q_{5}$} & $L_{5, \mathrm{j}}$ & $-Z_{0} X_{2}^{\prime}$ & $-Z_{0} Y_{2}^{\prime}$ & $X_{0} X_{2}^{\prime}+Y_{0} Y_{2}^{\prime}$ \\
\cline { 3 - 6 } & $K_{5, \mathrm{j}}$ & $Z_{1}^{\prime} X_{2}^{\prime}$ & $Z_{1}^{\prime} Y_{2}^{\prime}$ & $-\left(X_{1}^{\prime} X_{2}^{\prime}+Y_{1}^{\prime} Y_{2}^{\prime}\right)$ \\
\hline
\end{tabular}

Table 4. Formulas for calculating $K_{\mathrm{i}, \mathrm{j}}$ и $L_{\mathrm{i}, \mathrm{j}}$ for $\varphi, \tau, \psi$ system of angles.

\begin{tabular}{|c|c|c|c|c|c|}
\hline$i$ & $Q_{\mathrm{i}}$ & $\begin{array}{c}L_{\mathrm{i}, \mathrm{j}} \\
K_{\mathrm{i}, \mathrm{j}}\end{array}$ & $\mathrm{j}=1$ & $\mathrm{j}=2$ & $\mathrm{j}=3$ \\
\hline 1 & $Q_{1}$ & $K_{1, \mathrm{j}}$ & $Y_{2}^{\prime} P_{7}-Z_{2}^{\prime} P_{6}$ & $Z_{2}^{\prime} P_{5}-X_{2}^{\prime} P_{7}$ & $X_{2}^{\prime} P_{6}-Y_{2}^{\prime} P_{5}$ \\
\hline 3 & $Q_{3}$ & $K_{3, \mathrm{j}}$ & $-Z_{2}^{\prime} X_{1}^{\prime}$ & $-Z_{2}^{\prime} Y_{1}^{\prime}$ & $Y_{2}^{\prime} Y_{1}^{\prime}+X_{2}^{\prime} X_{1}^{\prime}$ \\
\hline \multirow{2}{*}{4} & \multirow{2}{*}{$Q_{4}$} & $L_{4, \mathrm{j}}$ & $Y_{0} \hat{P}_{7}-Z_{0} \hat{P}_{6}$ & $Z_{0} \hat{P}_{5}-X_{0} \hat{P}_{7}$ & $X_{0} \hat{P}_{6}-Y_{0} \hat{P}_{5}$ \\
\cline { 3 - 6 } & & $K_{4, \mathrm{j}}$ & $-\left(Y_{1}^{\prime} \hat{P}_{7}-Z_{1}^{\prime} \hat{P}_{6}\right)$ & $-\left(Z_{1}^{\prime} \hat{P}_{5}-X_{1}^{\prime} \hat{P}_{7}\right)$ & $-\left(X_{1}^{\prime} \hat{P}_{6}-Y_{1}^{\prime} \hat{P}_{5}\right)$ \\
\hline \multirow{2}{*}{6} & \multirow{2}{*}{$Q_{6}$} & $L_{6, \mathrm{j}}$ & $-Z_{0} \hat{P}_{1}$ & $-Z_{0} Y_{2}^{\prime}$ & $X_{0} \hat{P}_{1}+Y_{0} Y_{2}^{\prime}$ \\
\cline { 3 - 6 } & $K_{6, \mathrm{j}}$ & $Z_{1}^{\prime} \hat{P}_{1}$ & $Z_{1}^{\prime} Y_{2}^{\prime}$ & $-\left(X_{1}^{\prime} \hat{P}_{1}+Y_{1}^{\prime} Y_{2}^{\prime}\right)$ \\
\hline
\end{tabular}

\section{Results of numerical calculations}

The purpose of the experiments is to reveal the features of the application of the orientation angles systems of the shooting camera by comparing the root-mean-square errors in determining the spatial coordinates obtained by the method of space forward intersection, using all of the 5 listed systems of the angles of external orientation.

Systems of orientation angles in aerial survey: For experimental studies, a series of pairs of mock-up images corresponding to the conditions of aerial photography of a high-rise engineering structure from a multicopter unmanned aerial vehicle (UAV) were simulated. The diagrams in Fig. 1-2 show the results of a numerical experiment for 2 arbitrary pairs of images: RMS values introduced only by orientation angles. The following assumptions correspond to the experiment: flight altitude $100-110 \mathrm{~m}$; focal length of the shooting camera $f=35 \mathrm{~mm}$; pixel size pix $=5.6$ microns, matrices $36 \times 24 \mathrm{~mm}$; RMSE of determining orientation angles $m_{\mathrm{U}}=0.015^{\circ}$, RMSE of determining spatial position $m_{\mathrm{S}}=0.02 \mathrm{~m}$; RMSE of image measurement $m_{\mathrm{xy}}=0.5$ pix. The distance to the subject of photography is $50-80$ $\mathrm{m}$. The values of the orientation angles corresponded to an arbitrary shooting case. For example, the angles $\alpha, \omega, \chi$ for three images, from which two pairs of images were composed, had the corresponding values: $29^{\circ}, 75^{\circ}, 5^{\circ} ;-61^{\circ}, 6^{\circ}, 5^{\circ} ; 6^{\circ},-60^{\circ}, 4^{\circ}$.

From the diagrams (Fig. 1-2) it follows that RMSE of determining spatial coordinates for 
each system of orientation angles are different. The least preferred system of angles in this experiment is the one of aviation angles, for which the total RMSE

$$
M_{\mathrm{U}}=\sqrt{M_{\mathrm{XU}}^{2}+M_{\mathrm{YU}}^{2}+M_{\mathrm{ZU}}^{2}}
$$

has the greatest value. The smallest value of RMSE corresponds to: systems of angles $\alpha, \omega$, $\chi$ and $\bar{\omega}, \varphi, \kappa$ in pair 1 ; system of angles $\bar{\omega}, \varphi, \kappa$ in pair 2 . For the systems of angles $\Omega, J$, $\omega$ and $t, \alpha_{0}, \chi$, the value of $M_{\mathrm{U}}$ is actually the same in pair 1 and in pair 2 . The numerical experiment also allowed to reveal that the contribution of RMSE $M_{\mathrm{XU}}, M_{\mathrm{YU}}, M_{\mathrm{ZU}}$, caused only by errors in the orientation angles, to the RMSE of determining the spatial coordinates $M_{\mathrm{X}}, M_{\mathrm{Y}}, M_{\mathrm{Z}}$, which are the result of the influence of all parameters of the error model [9], is significant and amounts to $60-80 \%$.

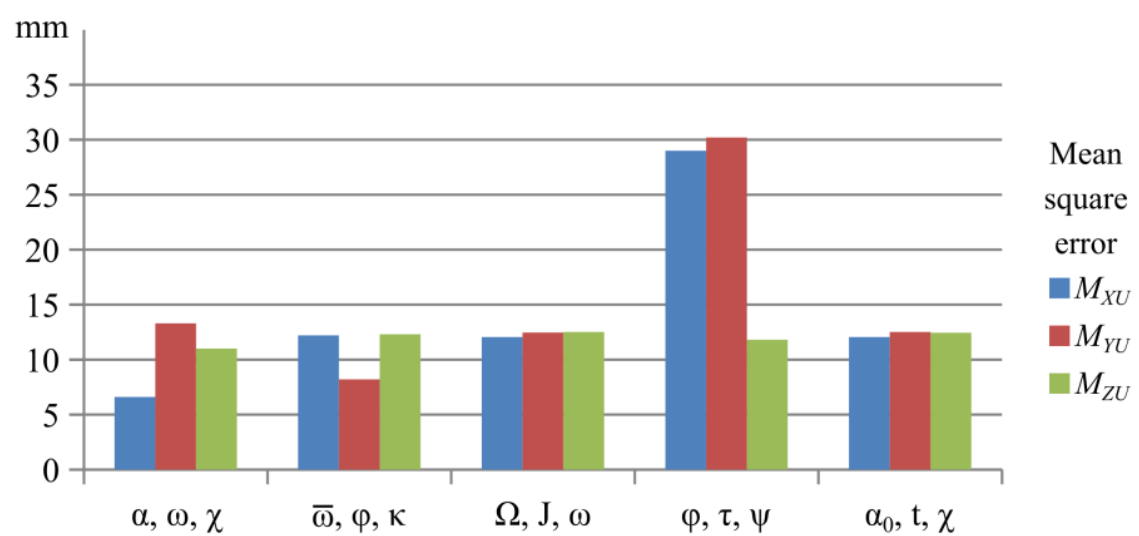

Fig. 1. UAV: RMSE for different systems of orientation angles, pair 1.

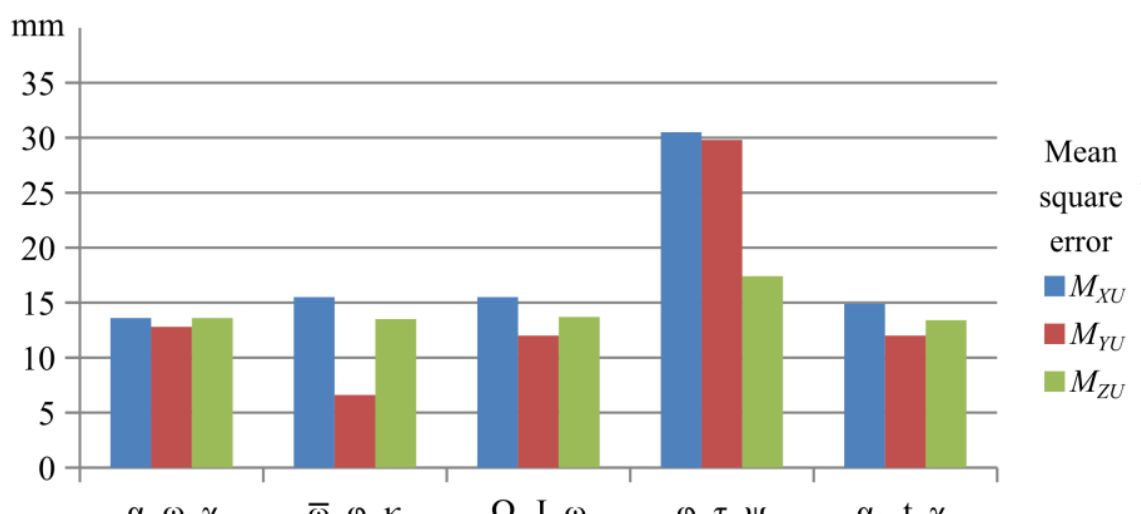

$$
\alpha, \omega, \chi \quad \bar{\omega}, \varphi, \kappa \quad \Omega, J, \omega \quad \varphi, \tau, \psi \quad \alpha_{0}, \mathrm{t}, \chi
$$

Fig. 2. UAV: RMSE for different systems of orientation angles, pair 2.

Systems of orientation angles in space survey. For experimental studies, a series of pairs of mock-up images belonging to one orbit and allowing one to determine the spatial coordinates of points on the Earth's surface for different latitude and longitude, and also to calculate the RMSEs corresponding to these coordinates, were simulated. The diagrams in Fig. 3-6 show the results of a numerical experiment, which corresponds to the following assumptions: 
- orbital altitude above the Earth $H=475 \mathrm{~km}$, orbital inclination $J_{\mathrm{OR}}=97.3^{\circ}$;

- focal length of the shooting system $f=4000 \mathrm{~mm}$, pixel size pix $=6$ microns;

- RMSE of determining the angles of orientation of the Earth remote sensing spacecraft (ERS SC) $m_{\mathrm{U}}=0.2^{\prime \prime}$;

- RMSE of determining the spatial position of the ERS SC $m_{\mathrm{S}}=0.5 \mathrm{~m}$;

- RMSE of image measurement $m_{\mathrm{xy}}=0.5$ pix;

- convergence angle $35^{\circ}$.

The diagrams (Fig. 3-6) show RMSE contributed only by the orientation angles to each spatial coordinate and the contribution (in \%) of this RMSE to the one of determining the spatial coordinates of some points on the Earth's surface $M_{\mathrm{X}}, M_{\mathrm{Y}}, M_{\mathrm{Z}}$ located at latitude $(B)$ : $80^{\circ}, 52^{\circ}, 20^{\circ}, 5^{\circ}$. The values of RMSE correspond to a circuit.

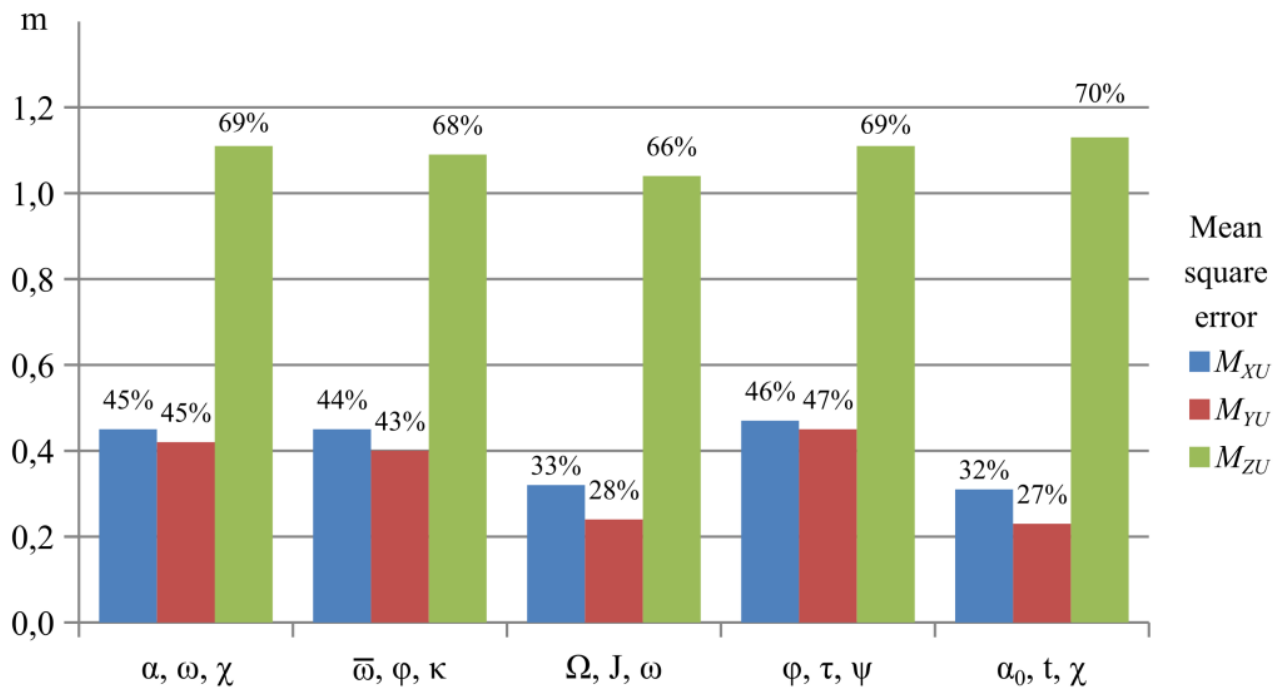

Fig. 3. ERS $\left(J_{\mathrm{OR}}=97.3^{\circ}\right)$ : RMSE for different systems of orientation angles, $B=80^{\circ}$.

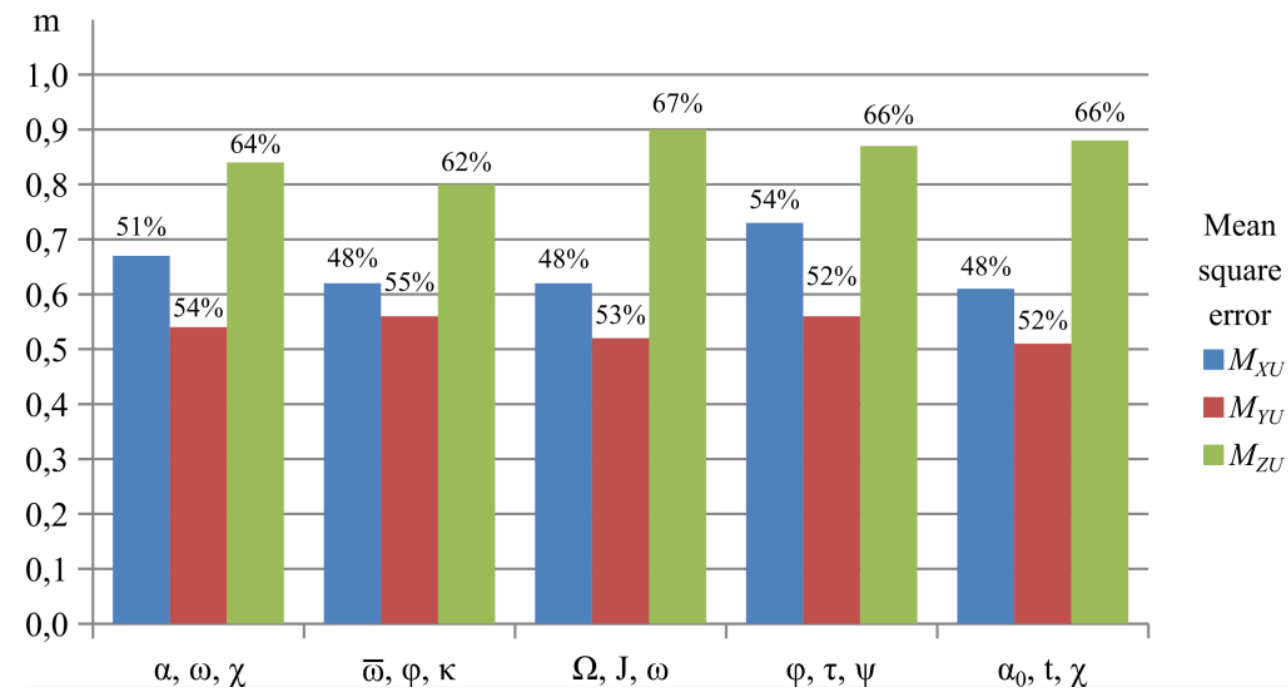

Fig. 4. ERS $\left(J_{\mathrm{OR}}=97.3^{\circ}\right)$ : RMSE for different systems of orientation angles, $B=52^{\circ}$. 


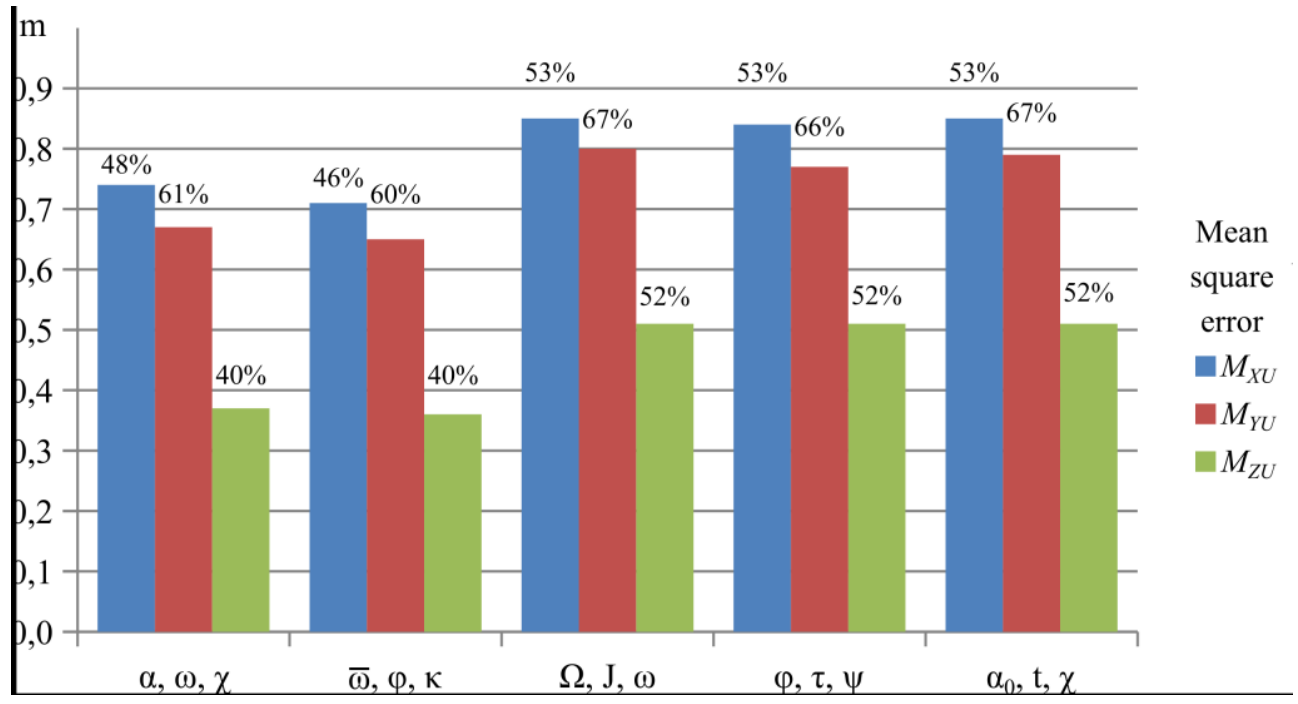

Fig. 5. ERS $\left(J_{\mathrm{OR}}=97.3^{\circ}\right)$ : RMSE for different systems of orientation angles, $B=20^{\circ}$.

From the diagrams (Fig. 3-6) it could be seen that RMSE of determining spatial coordinates for each system of orientation angles are different. The contribution of RMSE made by the orientation angles to the final RMSE of spatial coordinates $M_{\mathrm{X}}, M_{\mathrm{Y}}, M_{\mathrm{Z}}$ is also different. The dependence of RMSE for different coordinates on the latitude of the determined point is quite obvious and this is confirmed in the diagrams. At large latitude values, the $Z$ coordinate is determined with a larger error, i.e. worse. For small latitudes, the situation is reversed and the $X$ and $Y$ coordinates are determined worse. This is explained by the geometric conditions of the space forward intersection, the position of the base of the space survey relative to the axes of the coordinate system, in which the position of the ERS spacecraft and coordinates from the object under study are determined $[8,11,12]$. The analysis of $M_{\mathrm{U}}$ - total RMSE (8) - allows us to note that $M_{\mathrm{U}}$ has the smallest values: for systems of angles $\alpha, \omega, \chi$ and $\bar{\omega}, \varphi, \kappa$ at latitudes $20^{\circ}$ and $5^{\circ}$; for systems of angles $\Omega, J, \omega$ and $\alpha_{0}, t, \chi$ at latitudes $72^{\circ}$ and $80^{\circ}$. At latitude of $52^{\circ}$, the influence of all systems of orientation angles is actually equal, since the value of $M_{\mathrm{U}}$ for them is: $\min =1.2 ; \max =-1.3$ m.

For the purpose of a more complete analysis, the situation of determining the spatial coordinates and the corresponding RMSE for the same point was simulated using orbits with different inclination angles $\left(J_{\mathrm{OR}}\right)$, with the other parameters remaining unchanged in the experiments. The diagram (Fig. 7) for an orbit with an inclination $J_{\mathrm{OR}}=55^{\circ}$ shows the values of the RMSE and the contribution of errors from the orientation angles to the final RMSEs $M_{\mathrm{X}}, M_{\mathrm{Y}}, M_{\mathrm{Z}}$ for a point with latitude $B=52^{\circ}$ (longitude $L=35.5^{\circ}$ ) used in the previous experiment with an orbital inclination $J_{\mathrm{OR}}=97.3^{\circ}$ (Fig. 4). The total RMSE $M_{\mathrm{U}}$ (Fig. 7) has the smallest values for the systems of angles $t, \alpha_{0}, \chi$ and $\Omega, J, \omega$. For these systems of angles, a decrease in RMSE (by $28 \%$ ) can be noted, while for other systems of angles, an increase in RMSE is observed compared to the case when $J_{\mathrm{OR}}=97.3^{\circ}$ and where the total RMSE $M_{\mathrm{U}}(8)$ has the smallest values for systems of angles $\bar{\omega}, \varphi, \kappa$ and $\alpha_{0}, t, \chi$. 


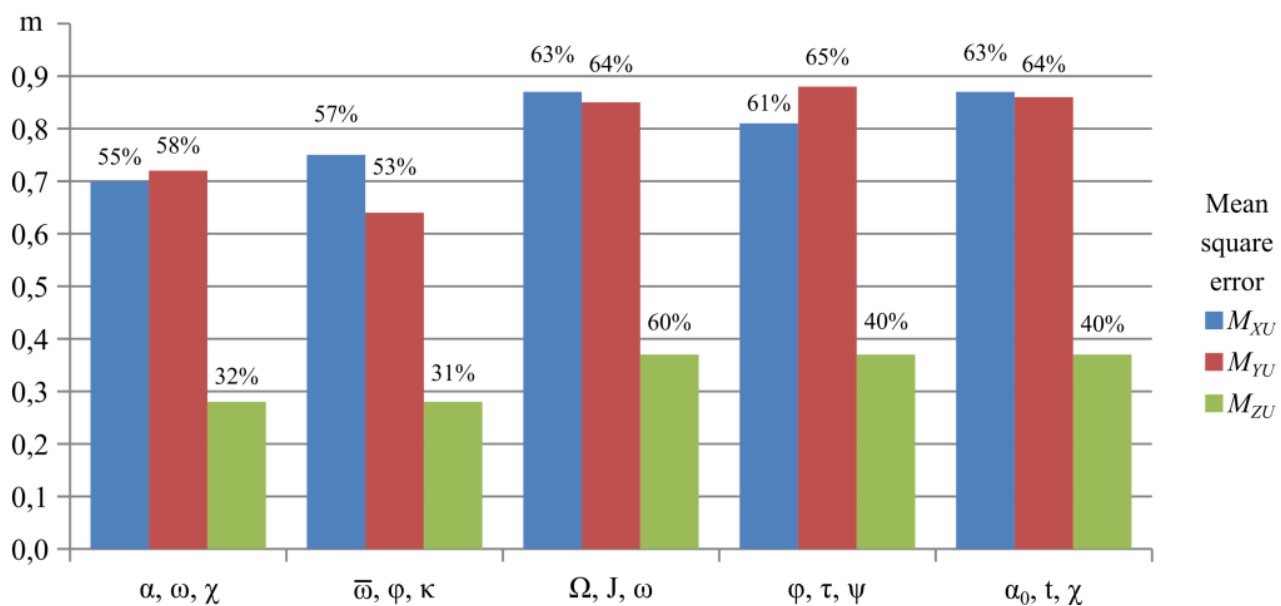

Fig. 6. ERS $\left(J_{\mathrm{OR}}=97.3^{\circ}\right)$ : RMSE for different systems of orientation angles, $B=5^{\circ}$.

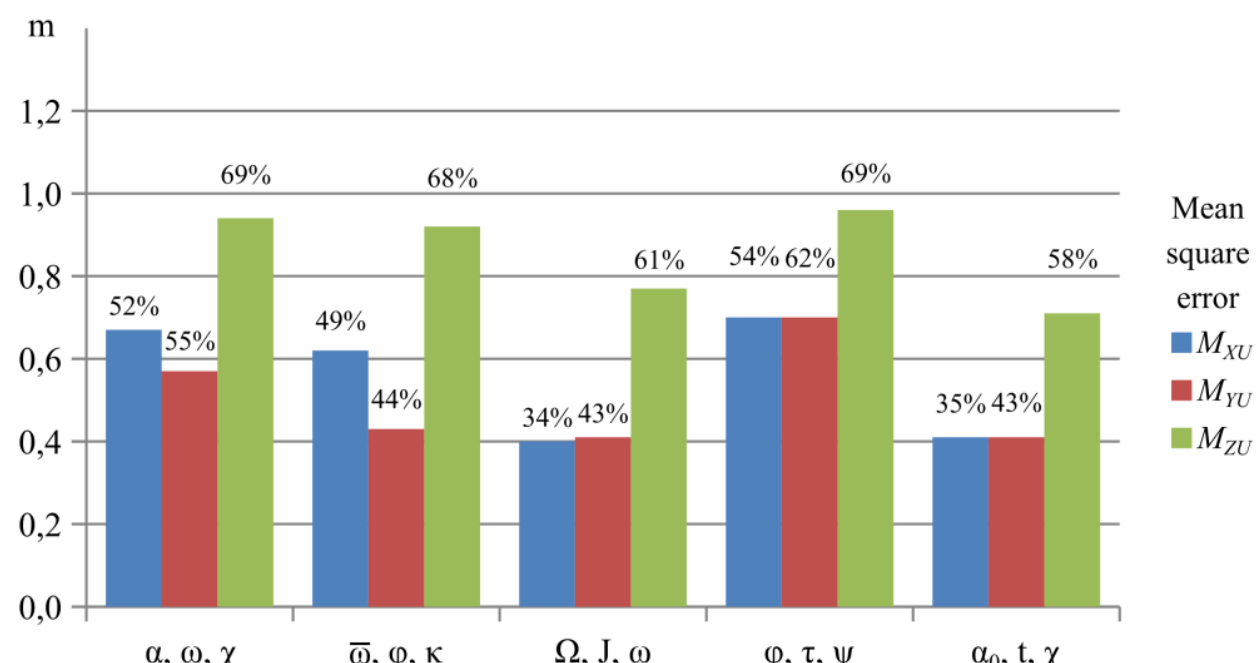

Fig. 7. ERS $\left(J_{\mathrm{OR}}=55^{\circ}\right)$ : RMSE for different systems of orientation angles, $B=52^{\circ}$.

\section{Conclusion}

The article presents the results of research aimed at identifying the features of using the systems of orientation angles of the shooting camera. The studies are based on the use of space forward intersection and the mathematical model of errors in determining the spatial coordinates built for it for the general case of survey [9]. The solution to this problem was expanded to five systems of angular elements of external orientation of the shooting camera. Studies carried out for five systems of exterior orientation angles allow us to draw the following conclusions:

The error in determining the spatial coordinates depends on the used angular orientation system. It is advisable to choose the angular orientation system taking into account the position of the shooting camera relative to the external coordinate system of the terrain or the coordinate system of the photographed object. This is true both in the case of aerial survey, for example, from an UAV, and in the case of space survey, when the orientation 
angles are large. The results of the numerical experiment simulating a survey from an ERS spacecraft having a polar orbit made it possible to establish that when processing space survey materials using a direct geocentric coordinate system, it is advisable to use: systems of angles $\Omega, J, \omega$ or $\alpha_{0}, t, \chi$ - for latitudes greater than $70^{\circ}$; systems of angles $\alpha, \omega, \chi$ or $\bar{\omega}$, $\varphi, \kappa-$ for latitudes less than $30^{\circ}$. The use of the complanarity condition, in comparison with the use of the collinearity condition, makes it possible to build a more comprehensive approach to identifying the influence of one or another system of angular orientation on the accuracy of determining spatial coordinates. Numerical simulation of RMSE of determining the spatial coordinates for various ERS spacecraft orbits, as well as orbital sections could be a tool for choosing the most suitable system of angles for processing georeferenced initial information (images).

The research results and the approach described in the article could be used to solve the problem of increasing the reliability of determining the spatial coordinates of the points of the object under study from the processing of its images.

\section{References}

1. I.T. Antipov, Geo-Siberia, 3, 52-58 (2006)

2. G. A. Avanesov, R. V. Besonov, A. N. Kurkina, A. A. Nikitin, A. A. Forsch, Sovr. Probl. DZZ Kosm, 16, 59-64 (2019)

3. V. N. Kolesnikov, O. I. Kozlov, Geodesy and aerophotosurveying, 62, 383-389 (2018)

4. R. N. Achmetov, V. V. Eremeev, A. E. Kuznetsov, G. N. Myatov, V. I. Poschehonov, R. N. Strayilayov, EES, 1, 44-53 (2017)

5. R. A Schovengerdt, RS.MMIP, USA (2007)

6. A. N. Lobanov, M. I. Burov, B. V. Krasnopevtsev, Fotogrammetriya, Russia (1987)

7. A. P. Mikhailov, A. G. Chibunichev, Fotogrammetriya, Russia (2016)

8. M. S. Urmaev Kosmicheskaya fotogrammetriya, Russia (1989)

9. V. M. Bezmenov, K. I. Safin, Geodesy and aerophotosurveying, 64, 415-422 (2020)

10. I. T. Antipov, MOAF, Russia (2003)

11. N. E. Zharova, A. G. Geodesy and aerophotosurveying, 6, 65-74 (2017)

12. N. E. Zharova, A. G. Geodesy and aerophotosurveying, 5, 79-86 (2017) 